\title{
AJVIL
}

\section{Assured PNT Through Multiple Diverse Technologies}

\section{Dr Paul D Groves}

Space Geodesy \& Navigation Laboratory University College London (p.groves@ucl.ac.uk)

ION GNSS+ 2016

14 September 2016

\section{UCL ENGINEERING}

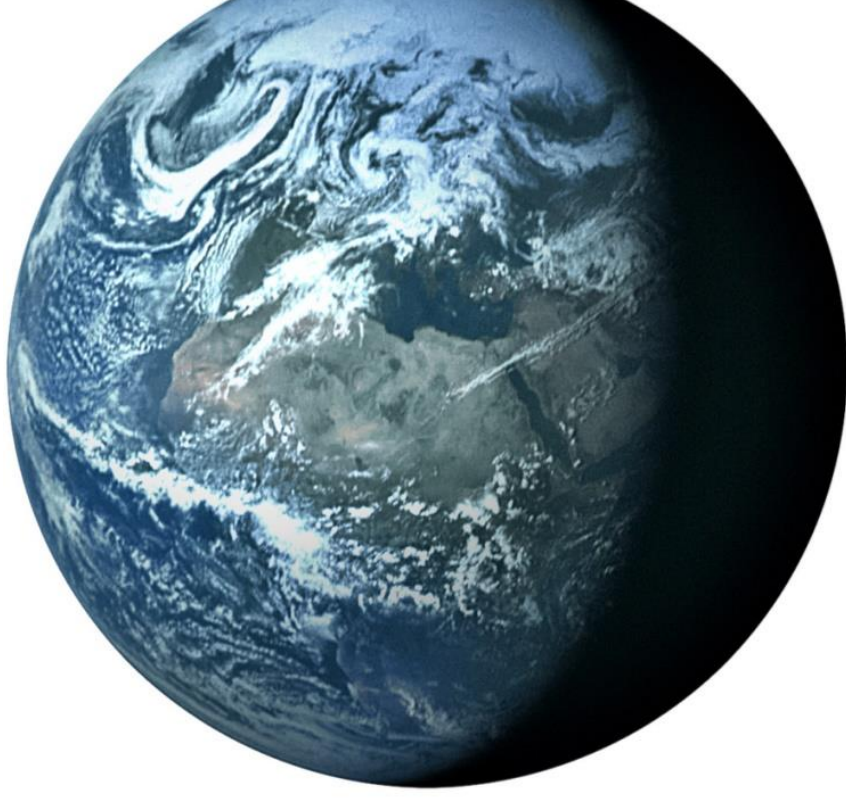




\section{Introduction}

"Panelists will have the opportunity to expound upon their vision of how ubiquitous, high-integrity PNT might be achieved"

\section{Ubiquitous means}

- Works in different environments

- Works for different user behaviours

- You always have a PNT solution

\section{CONFLICT}

\section{High-integrity means}

- You can always trust the PNT solution

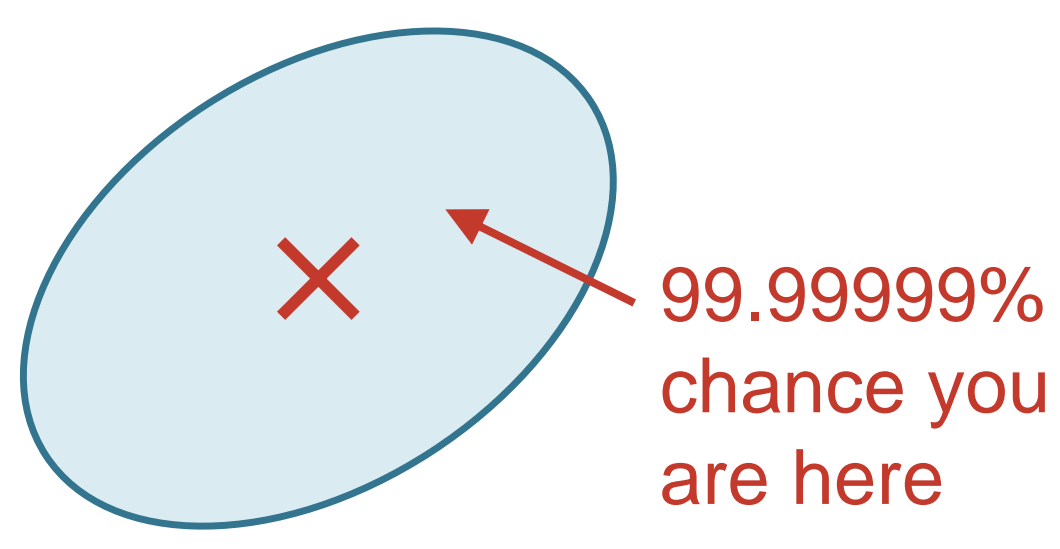

No solution is better than an untrustworthy solution 


\section{No Positioning Technology is Reliable}

GNSS and Other Radio Signals:

Jamming Spoofing Interference
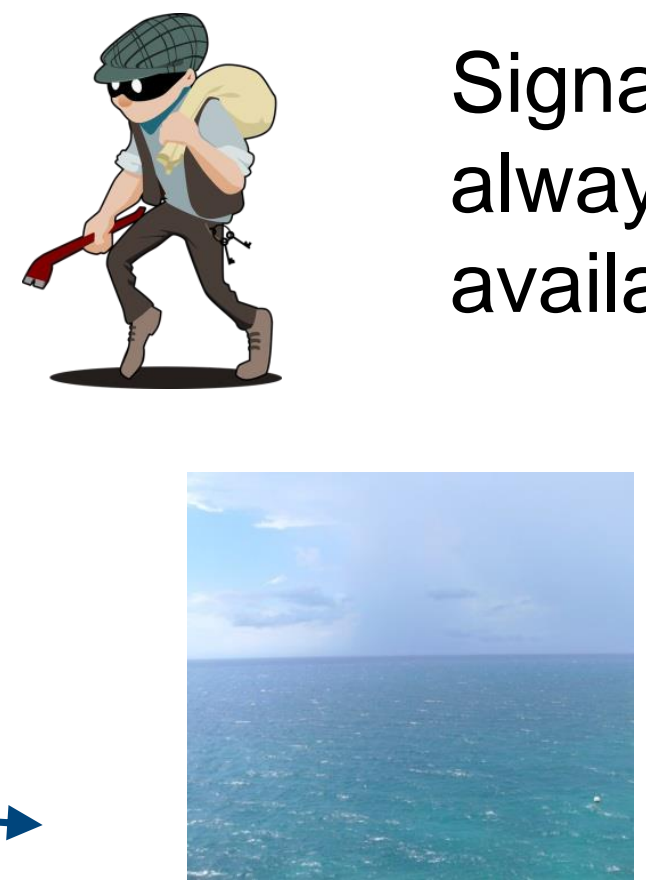

Dead Reckoning:

Errors grow with time
Signals not always available

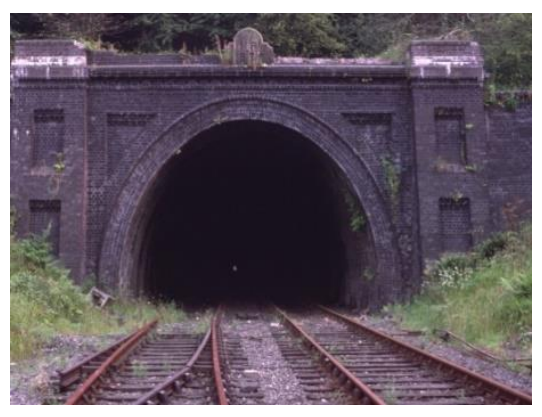

Visual Navigation: Landmarks are not available everywhere

Things Break:

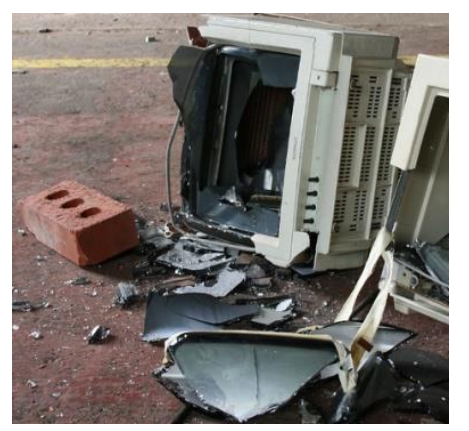




\section{Reliability Means Multiple Techniques}

Different navigation and positioning techniques must fail under different conditions

- Most radio positioning techniques perform poorly indoors

- Wi-Fi positioning works better indoors

- Low-frequency radio signals are difficult to jam or spoof

- LF signals also suffer from large propagation errors that must be calibrated

- Only dead-reckoning techniques provide continuous positioning

- Dead-reckoning position errors grow with time 


\section{What Do We Use When?}

$\geq 13$ smartphone pedestrian positioning techniques

Other platforms use other techniques

Focus processing resources on the most viable techniques

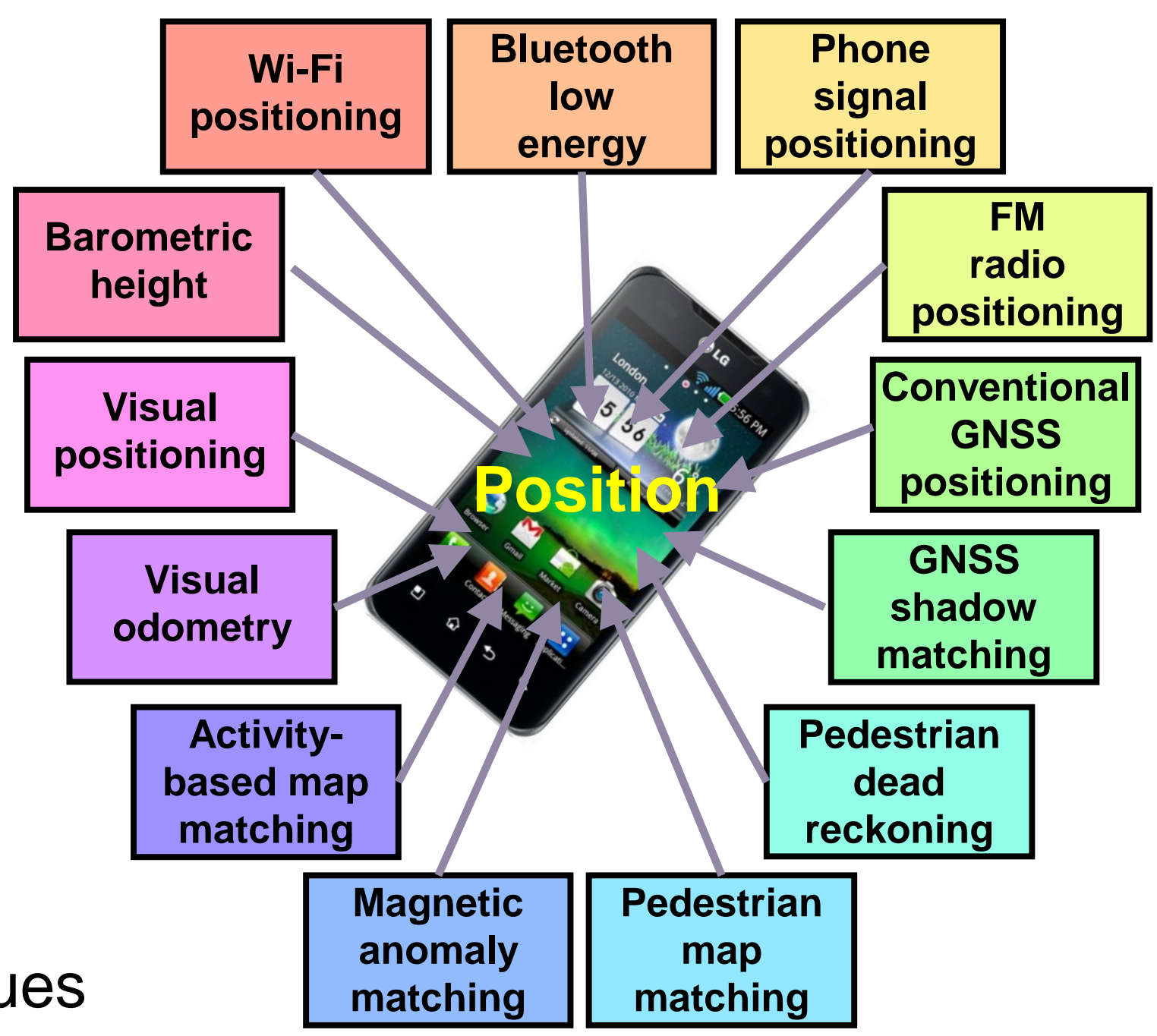




\section{What Do We Use When?}

It depends on the Context

\section{Environment}
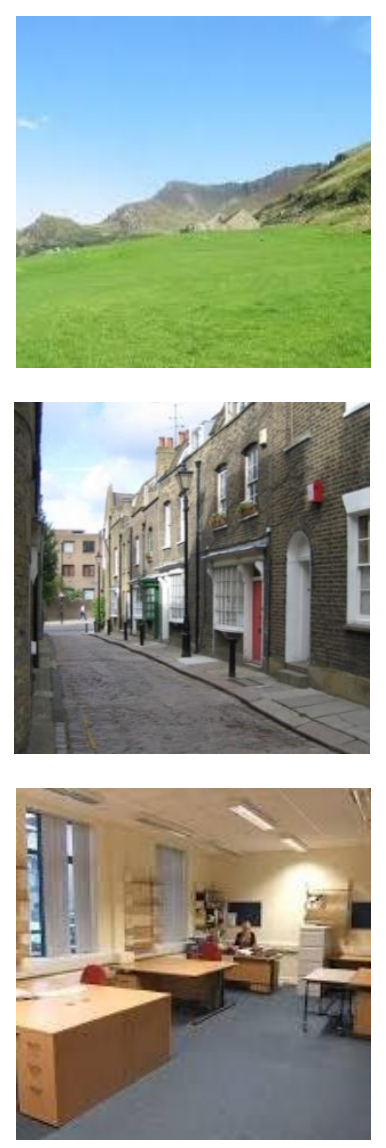

Urban: Use 3Dmapping aided GNSS

Open: Standard GNSS works well

\section{Indoor: Wi-Fi} generally best

\section{Behaviour}
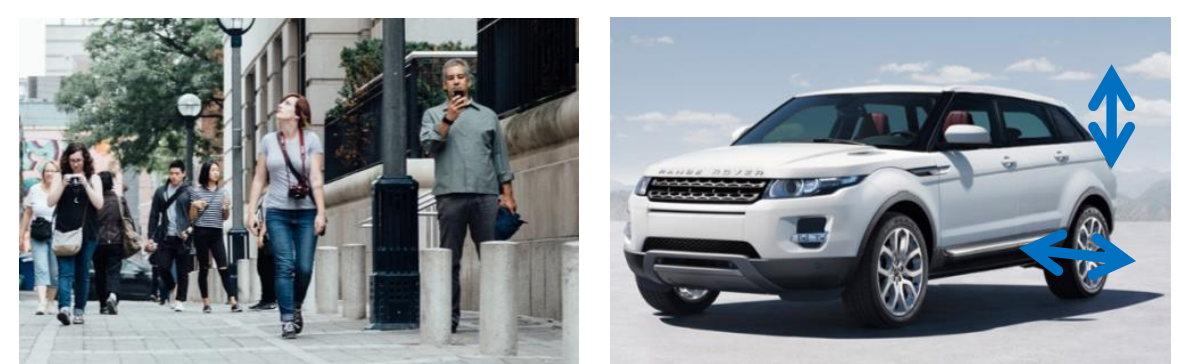

Pedestrians and Vehicles

- Different map matching

- Different motion constraints

- Step detection only works for pedestrians 


\section{Context-Adaptive Navigation}

We detect the environmental and behavioural context.

We select the appropriate navigation techniques

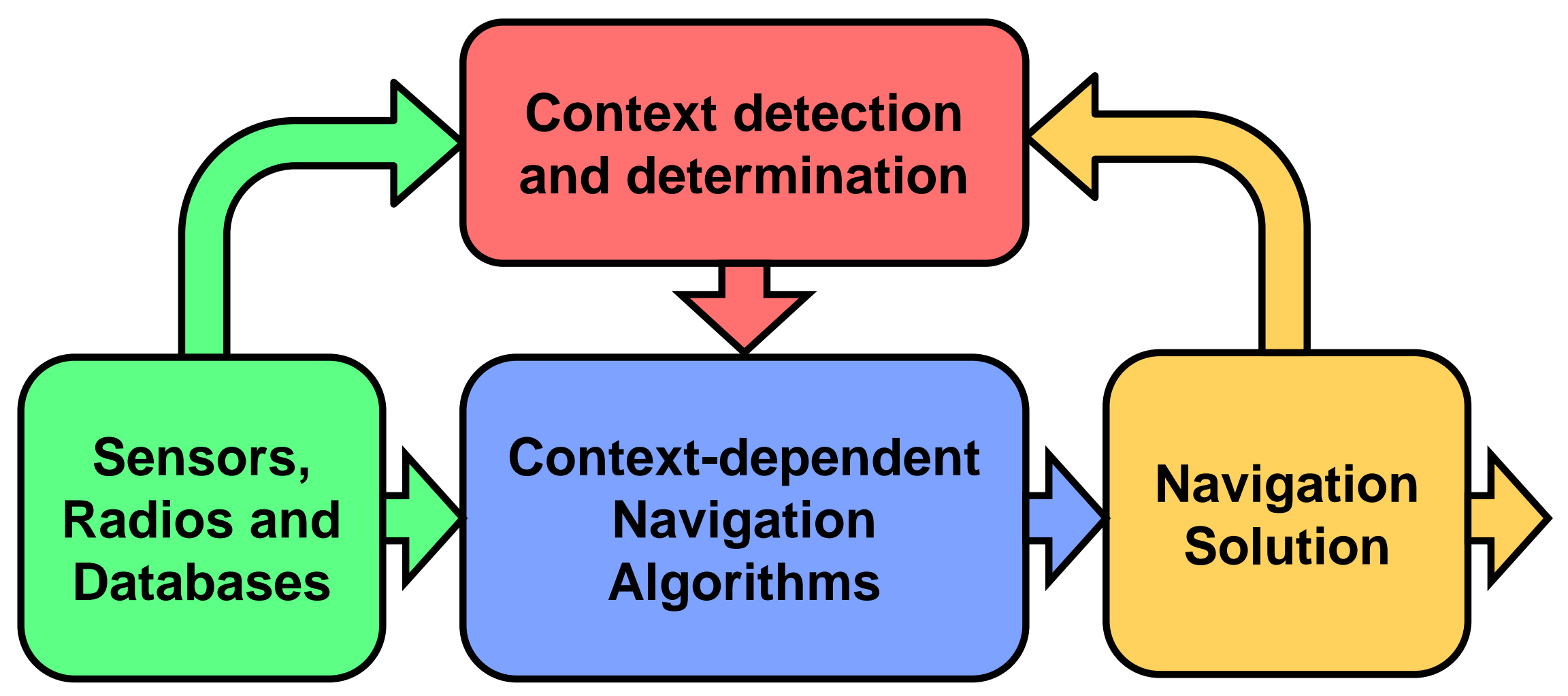




\section{What Can We Trust?}

Multisensor Navigation can ensure that something will always work
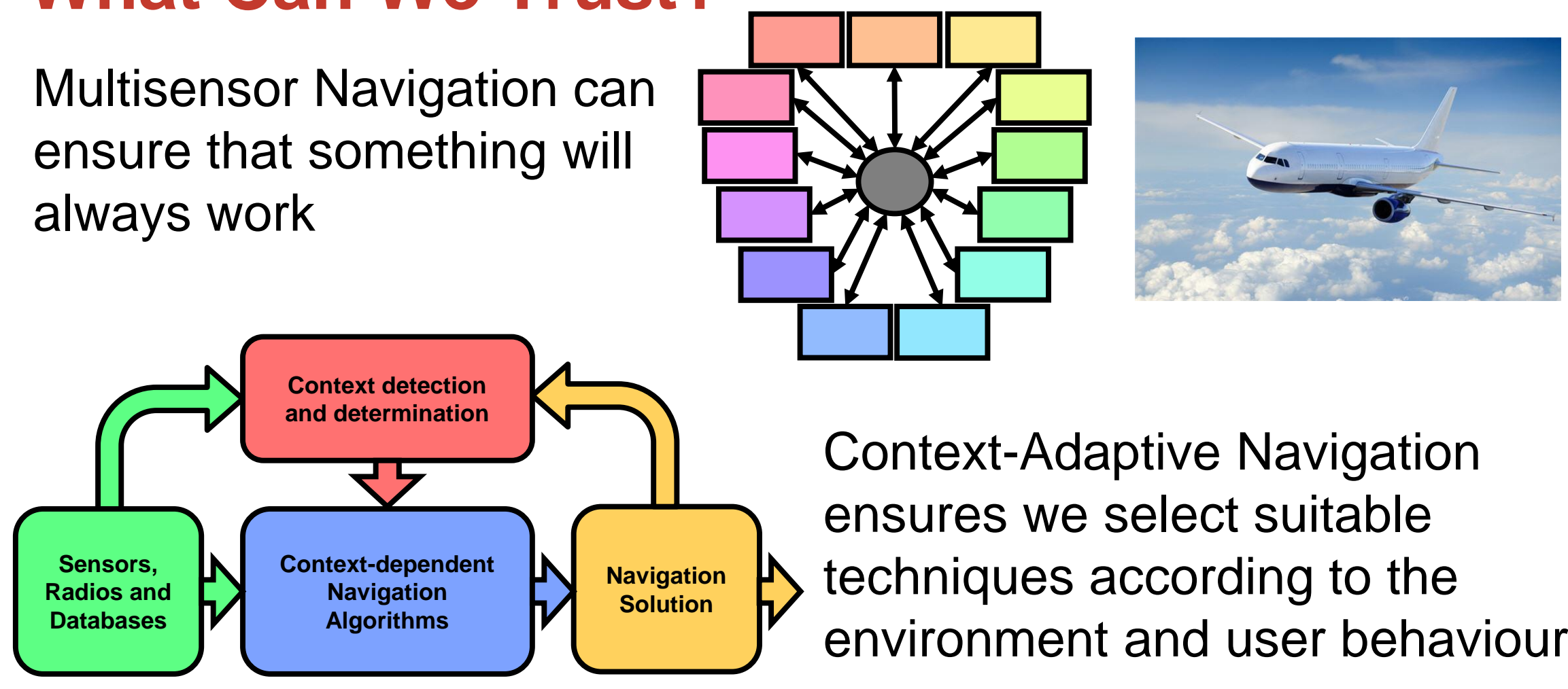

Context-Adaptive Navigation ensures we select suitable techniques according to the environment and user behaviour

BUT how do we know whether our position solution is right?
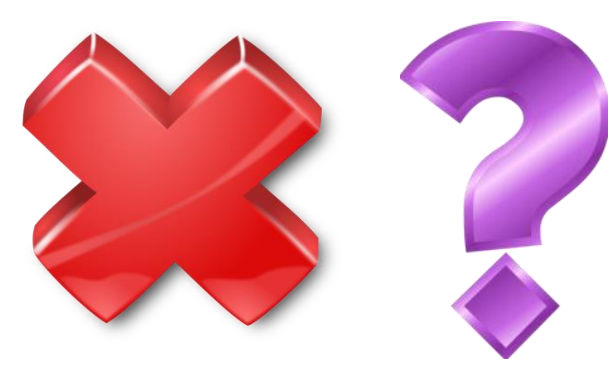


\section{We Need Solution Verification}

Measurements

Individual quality checks

Consistency checks - Does each measurement agree with a prediction made by the others?

Compute two independent solutions - Do they agree?

Can compare absolute position or change in position

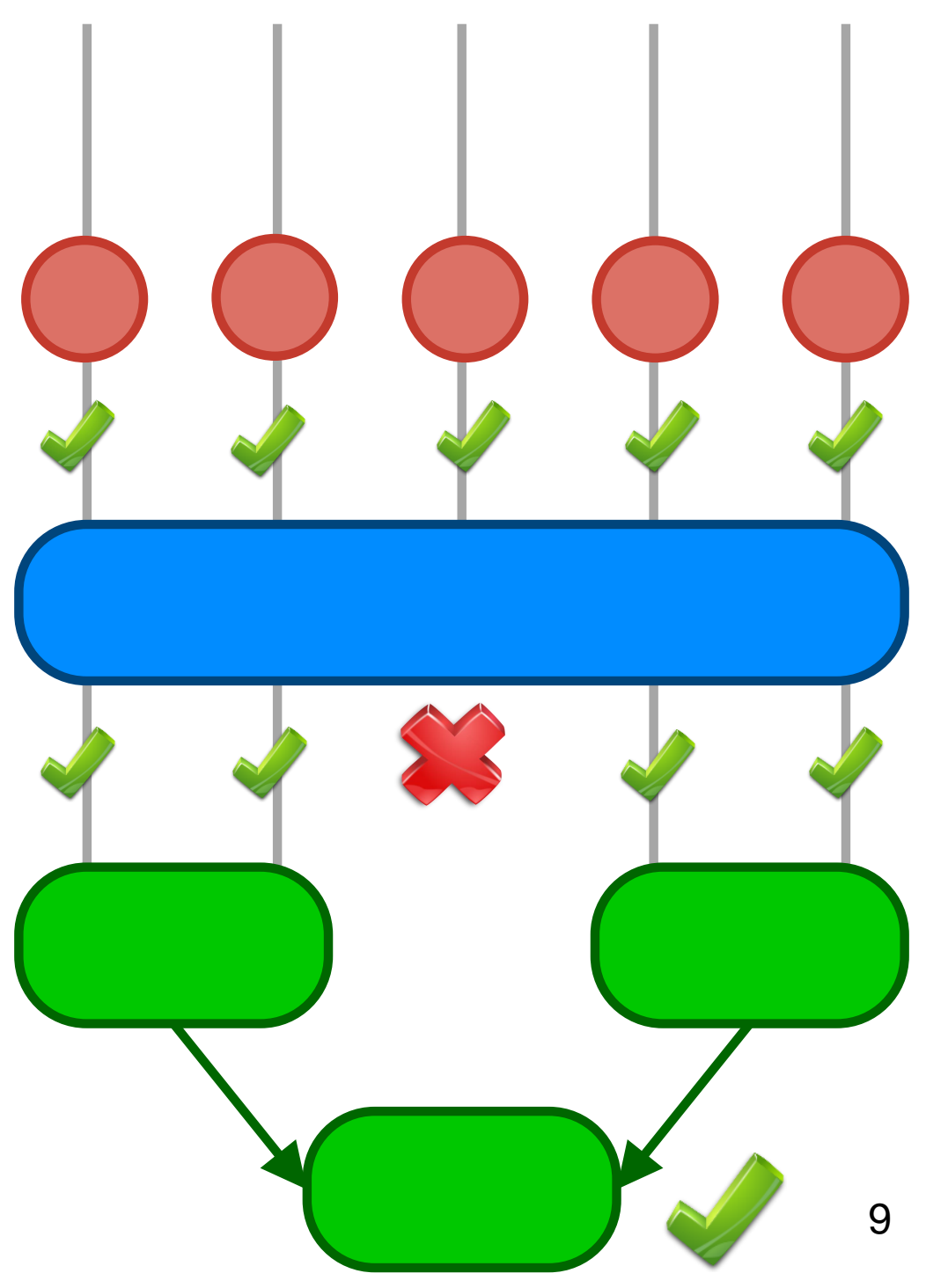




\section{The Complexity Challenge}

To combine and compare different navigation technologies we need to understand them

- Accuracy?

- Error characteristics?

- Correlation times?

- Failure modes?
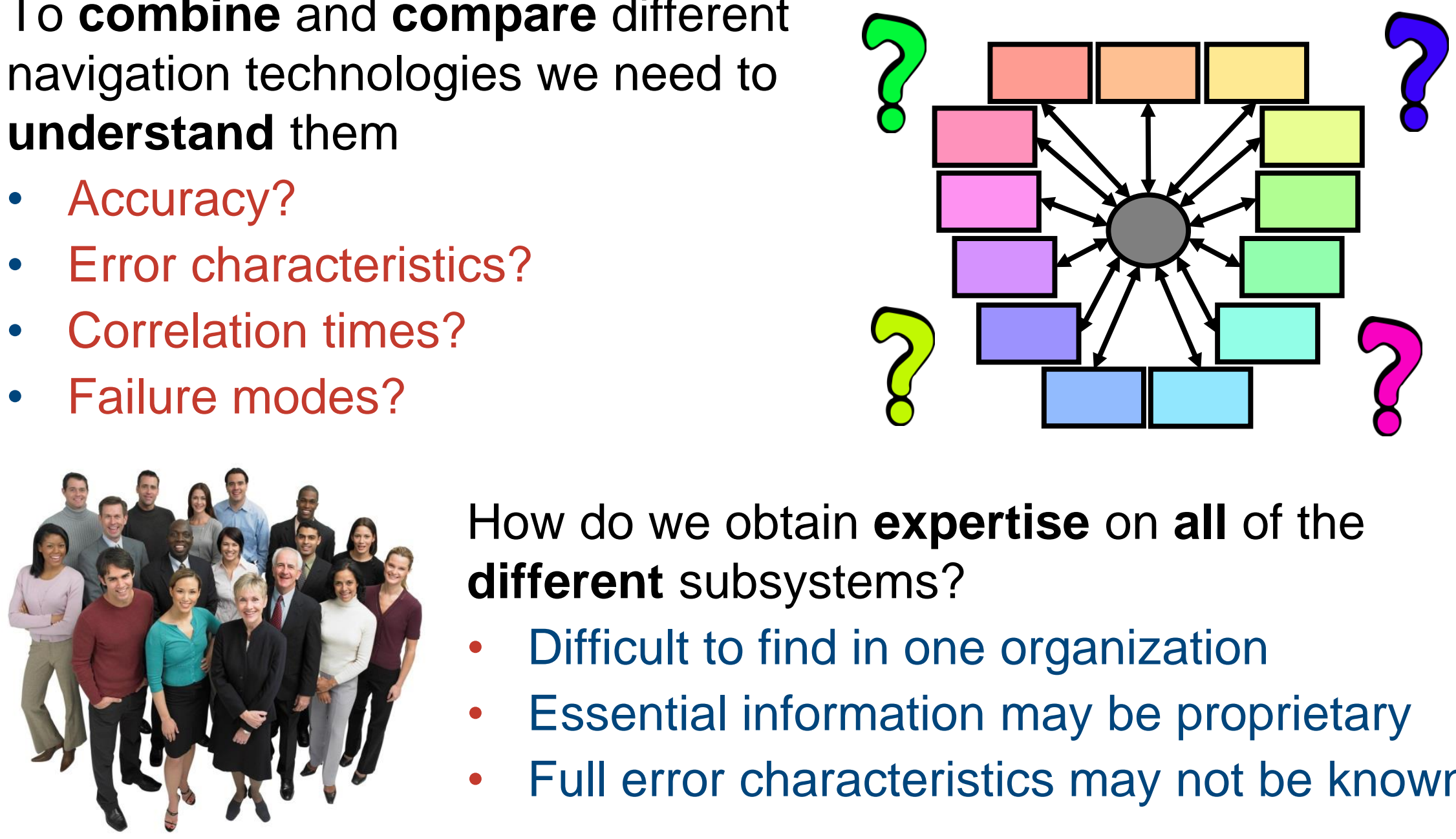

How do we obtain expertise on all of the different subsystems?

- Difficult to find in one organization

- Essential information may be proprietary

- Full error characteristics may not be known 


\section{The Plug 'N' Play Solution}

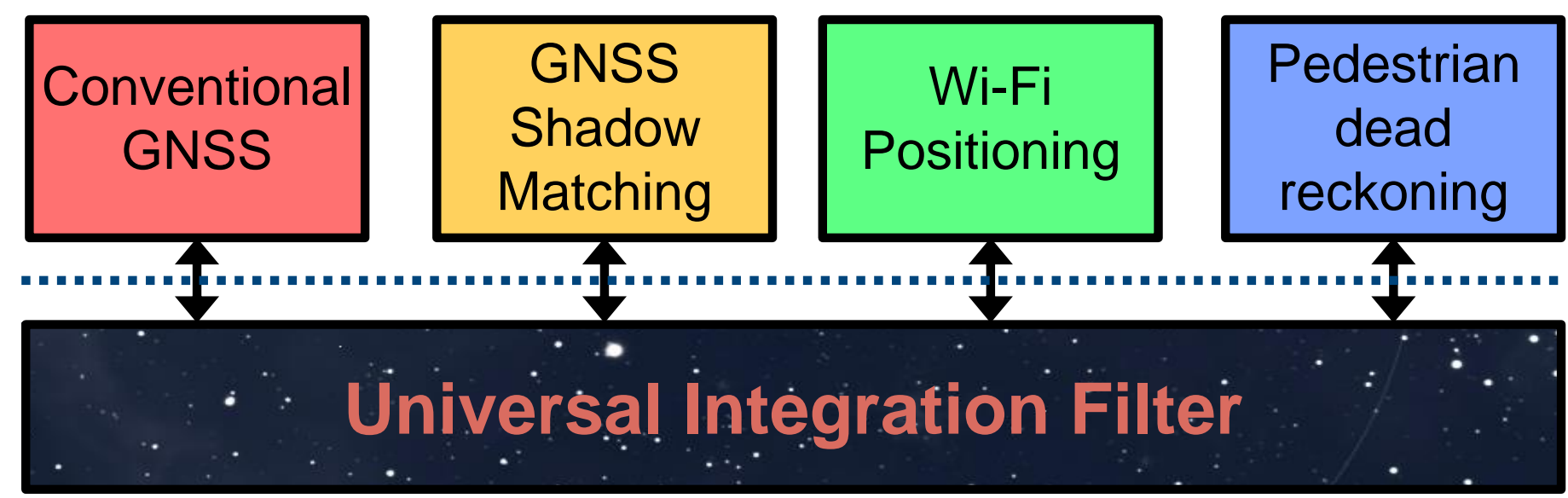

- Accepts several standard measurement types

- Configures automatically based on measurements received with sensor specifications

How do we trust the sensor specifications?

Certification Process? Expensive and time consuming

Learning Algorithms? Can we trust these? 


\section{Is a Standard Interface Realistic?}

How do we persuade everyone to conform to it?

Businesses compete to sell products and services

- Is there a big enough market for ubiquitous positioning and high integrity?

- Is it lucrative enough for them to share information with competitors?

Governments promote technologies that:

- Potentially create jobs

- Look good, e.g. satellite systems and "quantum" technology

BUT Are they interested in more reliable PNT? 


\section{Conclusions}

"Panelists will have the opportunity to expound upon their vision of how ubiquitous, high-integrity PNT might be achieved"

It is achievable technically:

1. Multiple PNT technologies with different failure modes

2. Context adaptivity

3. Multi-layered consistency-based verification

4. Plug ' $n$ ' Play integration with a standard interface

But, is it achievable politically?

Ubiquitous, high-integrity PNT needs cooperation 